\title{
Analysis of the Characteristics and Works of Traditional Guzheng Performance
}

\author{
Wang Xiaomei \\ Sichuan University of Science and Engineering Conservatory of Music, Sichuan, China, 643000
}

Keywords: kite; playing skills; playing style; genre

Abstract: Guzheng is an ancient plucked instrument in China. It has a history of more than 2,000 years. Its quaint style and unique national charm have always been regarded as one of the most traditional musical instruments with Chinese characteristics. In the long evolution of Guzheng, the evolution and development of traditional performance techniques have made the Jinle art have a more unique national charm and quaint style, and also make the zither technique a new level, both in appearance and technique. Both have undergone great reforms and innovations, forming their own unique playing style and performance system.

\section{Introduction}

Zheng, the ancient Chinese plucked instrument, has a history of more than 2,000 years. Its quaint style and unique national charm have always been regarded as one of the most traditional Chinese musical instruments. The pronunciation of the Zheng is bright and bright, and the sound is excellent. It is good at expressing the artistic conception and delicate euphemism, and can express the emotions of impassion and grandeur. Reviewing the history of the zither art that has passed, summing up the evolution of the zither performance techniques is especially important for the development of the zither music art.

\section{Development history of guzheng playing techniques}

During the Warring States period, Li Si wrote in his book "The Book of Deportation": "The husband fights and sings, and screams, and the voice of the true Qin is also." This is now The earliest documents found in the performance of Guzheng's performances, although there is no specific expression of performance techniques, we can conclude from the form of Guzhen's participation in performances that the original performance form of Guzheng and other national musical instruments is Singing accompaniment. The five major schools of guzheng formed in modern times, most of their representative works are derived from rap music and opera music. For example, Henan Zhengle, which is based on Henan Daqu, and Shandong Zhengle, which was developed on the basis of Shandong Qinshu. Tang was the heyday of economic and cultural development in ancient China, and it was also the prosperous period of the development of guzheng art. With the prosperity of Tang Daqu and music and dance, the Zheng also had great development in playing techniques[1]... Professor Jiao Wenbin's "Han Zheng History", a collection of more than 
one hundred poems related to the guzheng in "The Complete Tang Poetry", which recorded and described the performance techniques and fingerings of the Tang dynasty, including: cover, suppress, draw, dial Eighteen kinds, such as playing, shooting, curbing, pressing, swearing, appreciating, stroking, picking, squatting, pushing, playing, squatting, and maneuvering. Among them, four kinds of bullets, blocks, beats and dials are right-hand techniques, and the remaining twelve are left and right techniques. Judging from the application of these performance techniques, the performance technique of the Tang Dynasty Zheng has been quite complicated in the rhyme of the left hand, which greatly enriched the performance of the guzheng and increased the artistic effect. In modern times, in the various schools of Guzheng, most of them are right-handed and left-handed. This traditional technique of combining "sound" and "rhyme" is exactly what the guzheng style is. Guzheng training mode as shown below.

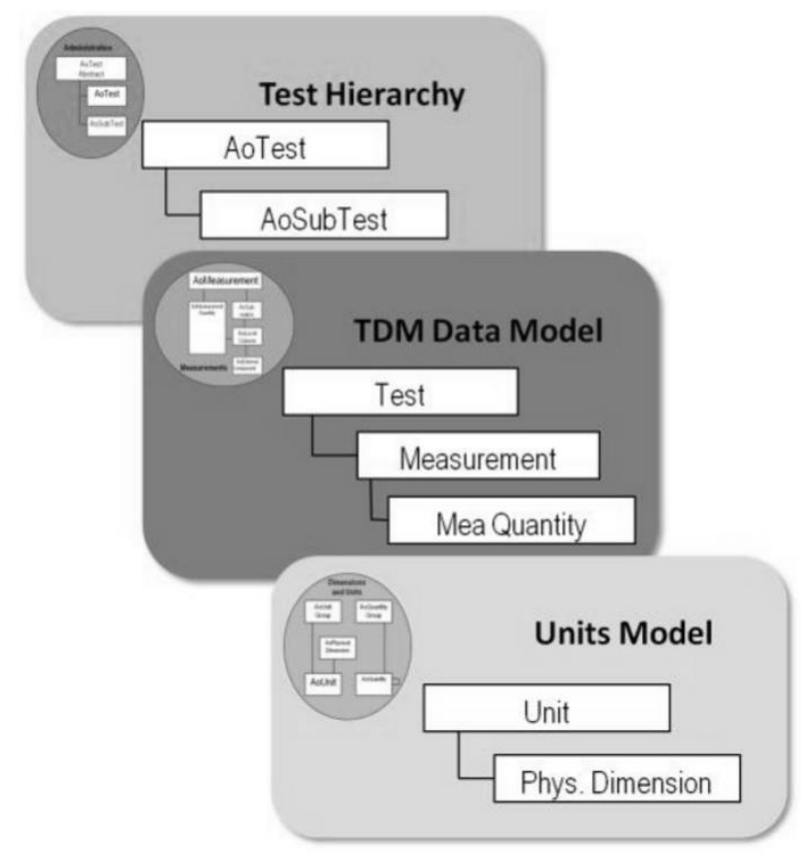

Fig.1 Guzheng training mode

\section{Development of modern guzheng playing techniques}

The second time, in the 1950s and 1960s, Mr. Wang Xizhi of the Zhejiang Zheng School created new performance techniques such as sweeping and fasting, and borrowed the Guqin technique, using double octaves of "double squats" plus sliding skills. Etc., greatly enriched the guzheng playing techniques, made up for the shortcomings of the guzheng lingering and the lack of fierceness, and laid a certain foundation for the guzheng to create a magnificent and intense scene of music. These techniques were used in his work "Lin Chong Night Run". Wang Xizhi's "Lin Chong Night Run" used a large section of "long shake" and "short shake" and "sweep"[2].

The traditional techniques of Zhepai, such as "point bomb", successfully expressed the musical image of the music and formed a style of grandeur and emotion. Wang Shuzhi's technological innovation not only enhanced the expressive power of Zheng music, but also enriched the singer writing techniques, and further promoted the development of zither skills.

For the third time, in the 1970s and 1980s, Mr. Zhao Manqin of Henan founded the "Quick Fingering Technique" system. This technique changes the single form of symmetry playing with only three fingers in the past, adopting four fingers for sequential and cyclical playing, so that the fingering combination of the guzheng forms a geometric number, expands the field of guzheng 
playing, and adopts the law of finger movement. The study of the playing method has improved the flexibility of the finger, enabling the guzheng to freely play the fast melody of the pentatonic, seventh-order and varying scales. The successful application of "Quick Finger Order" in "The Sun Red on Jinggang Mountain" and "Tigers Going Up the Mountain", especially the use of "Quick Finger Order" by Wang Zhongshan in "Yunling Sound Painting" played to the extreme The rapid fingering technique has been widely promoted throughout the country.

After the 1980s, the Guzheng community attracted a group of professional composers to participate in the creation and emerged a number of outstanding works. For example, Wang Jianmin's "Western Regions with Thoughts", Zhou Yiguo's "Yunshang v." and so on. These composers boldly broke the original way of setting, created a new creative idea, promoted the progress of playing skills, and developed many new techniques[3].Guzheng training data as shown below.

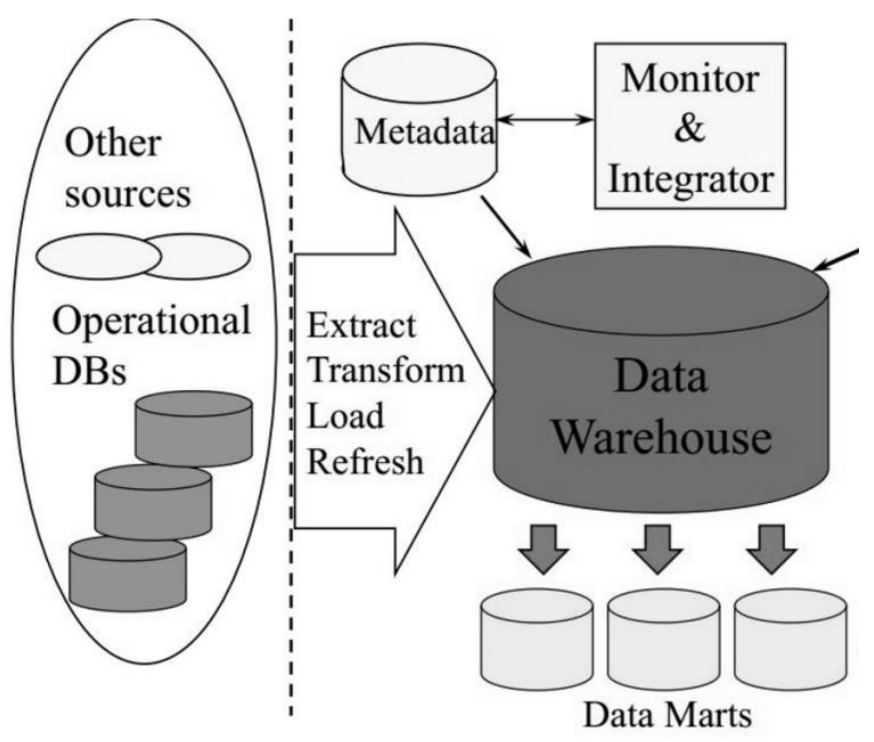

Fig.2 Guzheng training data

From the first time in 1955, "Golden Year", the simple use of left-handed playing techniques to the "Yunling Sound Painting" (Wang Zhongshan) left hand complex sound, round, and other difficult performance techniques, just a few decades The development of guzheng techniques has far exceeded the previous two thousand years. The "liberation of the left hand", breaking the traditional application of the "three-finger" to the "fast fingering technique system" of the right hand, added the expression charm of the traditional instrument of the guzheng, and made great contributions to the integration of the zheng music into the national music.

\section{Guzheng performance analysis}

\subsection{Change of order}

The traditional techniques of the right hand of Zheng music are mainly the use of hook, wipe, and support. The techniques of Guzheng, with the development of the times, draw on and absorb the skills of other instruments to enrich their expressiveness. Modern kites often arrange the order according to the rules of the melody. The fingers are no longer limited to the three fingers of the right hand, the index finger and the middle finger, but are used by both hands to emphasize the independence of the fingers. They often break in the creation of modern zither music. Guzheng traditional pentatonic arrangement, seven-level scale and various special scales arranged in many 
tracks, so the traditional octave string can not meet the needs of modern kites.

Special sound sequences and fast and varied melody are carried out, and Mr. Zhao Manyin's "Quick Fingering Technique" system solves this problem.

\subsection{References to other instruments playing skills}

The voice technology is a new skill formed by the harp and piano. The vocal performances in modern zither music have exceeded the limitations of three or four sounds, especially the seven consecutive sounds of the left hand, requiring interlude tone, balance of force points, and coherence.

Of course, Guzheng also has a lot of skills to draw on other instruments, such as the more than two octaves formed by the sound of the antique piano, and the double wiping of the dulcimer, which has enriched the expressive power of the guzheng to a certain extent.

\subsection{Development and application of left-hand techniques}

The participation of the left hand can be said to be the biggest difference between the modern zither technique and the traditional zither technique. In addition to the original syllabic function, the left hand also began to perform sub-finger, chord, phonological accompaniment and other auxiliary performance techniques. Play the music melody theme. The left-handed playing technique has thus entered a new historical period[4].

\subsection{Development and application of other techniques}

In the creation of modern zither music, in order to achieve special rhythm and sound effects, the chord and other slap techniques were also innovated.

Such as: the singer of the singer "Theatrical Rhyme" (Wang Jianmin), in order to express the taste of "singing, doing, reading, playing" and reading the "play" in Chinese drama, using the slap

, slap the piano cover, playing on the left side of the piano code, no fixed pitch performance, elbow pressure string, fingertip slamming the piano board and other techniques. These techniques are performed in the zither songs such as "Huangling Thoughts", "Fantasia" and "Mountains' Desires".

\section{Thoughts on the development of kites}

The foundation of modern kite music is solid and colorful traditional kites. After thousands of years, under the influence of geography, language, customs and different art forms, traditional kites have formed various genres in the development: the simple and simple Shandong Zheng School, the charming Chaozhou Zheng School, and Gao Song. The rough Henan Zheng School, the lively and bright Zhejiang Zheng School, and the delicate and elegant Hakka School.

\section{Summary}

The development of the technique of kite music from one hand to two hands, from the appearance of the finger to the application of the rapid finger order, all add to the charm of the traditional instrument of guzheng, which is also the result of the evolution of the guzheng in the long process of technology. . However, when the development of modern zither technology is more direct in the performance of sci-fi, how to develop on the basis of maintaining the original style of the instrument becomes the main problem we face. 


\section{References}

[1] Erica Bisesi,Richard Parncutt. An Accent-Based Approach to Automatic Rendering of Piano Performance: Preliminary Auditory Evaluation [J]. Archives of Acoustics, 2011, 36(2).

[2] Kosuke Itoh,Yukihiko Fujii,Kiyotaka Suzuki,Tsutomu Nakada. Asymmetry of parietal lobe activation during piano performance: a high field functional magnetic resonance imaging study [J]. Neuroscience Letters, 2001, 309(1).

[3] Werner Goebl,Caroline Palmer. Tactile feedback and timing accuracy in piano performance [J]. Experimental Brain Research, 2008,186(3).

[4] Wei LI. Humanistic Quality, Musicality, and Performance Skills in Piano Performance [J]. Cross-Cultural Communication, 2015, 11(3). 